\title{
The Silver Colloidal Solutions and Films for Efficient SERS
}

\author{
A. GórskA*, S. Meyer and A. Balter \\ Institute of Physics, Nicolaus Copernicus University, Grudziądzka 5/7, 87-100 Toruń, Poland
}

\begin{abstract}
The silver colloids and silver films are known for their signal enhancing properties in Raman spectroscopy. Surface enhanced raman spectroscopy is a very useful tool to detect molecules at low concentration or even single molecules in examined samples. Here we present a study on the preparation of silver colloidal solution and deposition of silver films on mica. The colloidal solutions were examined by two methods: the UV-VIS spectroscopy to determine their optical properties and atomic force microscopy to determine the size of the particles and structure of the deposited films. The crystal violet (tris(4-(dimethylamino)phenyl)methylium chloride) solution was used as a test sample for evaluation of enhancing properties of silver colloidal solutions and films. These experiments have shown that both colloidal solutions and films, are efficient surface enhanced raman spectroscopy substrates, therefore they can be used in further studies for enhancement of the Raman signal of biopolymers, f.e. polysaccharides and DNA.
\end{abstract}

PACS: 68.37.Ps, 78.30.-j, 82.70.Dd

\section{Introduction}

The Raman spectroscopy is a valuable tool for detection and characterization of molecules. Since the Raman signals are weak and do not allow one to investigate substances at low concentrations, signal enhancement techniques shall be applied. The surface enhanced Raman spectroscopyRS (SERS) technique is one of the possibilities, using metallic surfaces or nanoparticles as an enhancing substrate [1-4]. Nanostructures made of silver, gold, and copper are widely known as effective SERS substrates. The SERS technique has been used in recent years in many different research fields like biology, chemistry (mainly electrochemistry) and physics, both solid state and soft matter. The reported signal enhancement is up to $10^{14}$ for single molecule experiments [2]. The mechanism of the enhancement is not completely understood as yet. In general, two main contributions have been proposed: the electromagnetic and the charge transfer mechanism [5]. The surface enhanced resonance Raman spectroscopy (SERRS) allows one to obtain even larger enhancement and single molecule detection. The technique combines resonance Raman spectroscopy, operating in the maximum absorption range of the molecule of interest and enhancement caused by the presence of metallic structures. The enhancement can be characterized by few factors, in this work analytical enhancement factor (AEF) will be applied, defined as Eq. (1):

$$
\mathrm{AEF}=\frac{I_{\mathrm{SERS}} / c_{\mathrm{SERS}}}{I_{\mathrm{RS}} / c_{\mathrm{RS}}}
$$

where $I_{\mathrm{SERS}}$ is intensity of the SERS signal, $I_{\mathrm{RS}}$ - the intensity of the standard Raman signal, $c_{\mathrm{SERS}}$ and $c_{\mathrm{RS}}$ are concentrations of the solution used for the SERS and non-SERS measurements, respectively [6].

\footnotetext{
* corresponding author; e-mail: agorska@fizyka.umk.pl
}

The $\mathrm{Ag}_{\mathrm{col}}$ solutions and films (often called silver island films, SIFs) are widely used as an enhancing factor in many techniques, not only the Raman spectroscopy, but for example also in the fluorescence microscopy. Metal-enhanced fluorescence (MEF) has been observed for many hybrid systems composed mainly of very stable and highly fluorescing molecules, such as organic dyes and semiconductor nanostructures [7].

In this work we study the possibility of application of the $\mathrm{Ag}_{\text {col }}$ solution as an enhancing substrate in SERS experiments. We determined the absorption properties and diameter of the $\mathrm{Ag}$ particles in solution and used the $\mathrm{Ag}_{\mathrm{col}}$ solutions and films (silver island films, SIFs) as an enhancing substrate in SERS experiments conducted with crystal violet.

\section{Materials and methods}

\subsection{Ag colloidal solutions}

The $\mathrm{Ag}_{\mathrm{col}}$ solution were prepared by the Lee-Meisel method [8]. The basic procedure consisted of following steps: $90 \mathrm{mg}$ of $\mathrm{AgNO}_{3}$ was added into $500 \mathrm{ml}$ of pure water and heated up to $100^{\circ} \mathrm{C}$. The $10 \mathrm{ml}$ of $1 \%$ sodium citrate were added and the solution was boiled for $1 \mathrm{~h}$. In this way the $\mathrm{Ag}_{\mathrm{col}}$ solution of particles with the expected diameter of $40 \mathrm{~nm}$ was obtained.

\section{2. $U V-V I S$ and Raman spectroscopy}

Crystal violet $(\mathrm{CV})$ was chosen as a test sample for the Raman spectroscopy. It was obtained in its crystallized form and used to prepare solution in pure water $\left(10^{-4} \mathrm{M}\right)$. For both types of spectroscopy $1 \mathrm{ml}$ of the solution of interest was poured into a quartz cuvette. The $\mathrm{CV}$ and $\mathrm{Ag}_{\mathrm{col}}$ solutions were mixed, resulting in a final $\mathrm{CV}$ concentration of $5 \times 10^{-6} \mathrm{M}$. The $\mathrm{Ag}_{\text {col }}$ films were prepared on a mica surface, previously activated with a surfactant (cetyltrimethylammonium bromide (CTAB)) 
solution. A $25 \mu \mathrm{l}$ droplet of a mixture of $\mathrm{CV}$ and $\mathrm{Ag}_{\text {col }}$ was deposited onto a support and left in a desiccator for $24 \mathrm{~h}$.

UV-VIS spectra were recorded using a UV-VIS Specord M40 spectrometer (Zeiss, Germany). The Raman spectra was recorded by means of a modernized PHO double-grating Raman spectrometer (Coderg, France) using the $514.5 \mathrm{~nm}$ excitation line, equipped with a photomultiplier and a photon counter. The OriginPro 8 (OriginLab Corporation, USA) software was used for data processing.

\subsection{Atomic force microscopy}

For AFM imaging a $25 \mu \mathrm{l}$ sample of $\mathrm{Ag}_{\text {col }}$ solution was deposited onto a freshly cleaved mica surface and left in a desiccator for $24 \mathrm{~h}$. Dried samples were investigated using the Agilent 5500 AFM (Agilent Technologies, USA) in a contact mode. Software based image analysis was applied to all images taken (using PicoView 1.10.4. software from Agilent Technologies and SPIP 3.3.4.0 from Image Metrology, Denmark).

\section{Results}

\subsection{Determination of the properties of Ag solutions}

To determine optical properties of obtained $\mathrm{Ag}_{\text {col }}$ solutions, the UV-VIS spectra were recorded in order to extract the data about the maximum absorption region. The $\mathrm{Ag}_{\text {col }}$ exhibited an absorption maximum at $\lambda=419.5 \mathrm{~nm}$. The AFM imaging of 2 samples was per-

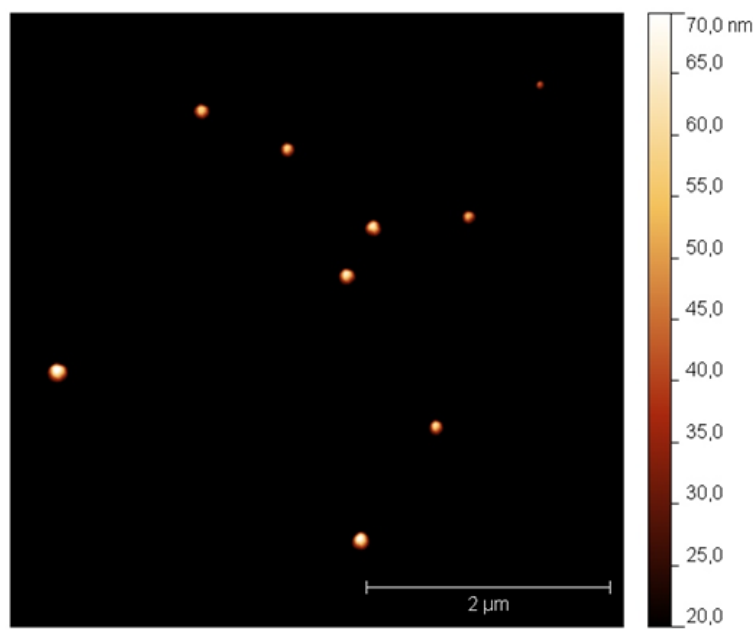

Fig. 1. A typical AFM image of $\mathrm{Ag}_{\text {col }}$ particles deposited on mica. The scan size is $5 \times 5 \mu \mathrm{m}^{2}$.

formed, for each sample 3 areas were investigated (3 images for each area of the sample). As a result 18 images were obtained and grain analysis in SPIP was performed (a typical image is shown in Fig. 1). The mean diameter of the particles in the $Z$ direction was $45 \pm 15 \mathrm{~nm}$, however particles of the diameter ranging from 20 up to $73 \mathrm{~nm}$ were observed. The histogram of the particles' diameter is shown in Fig. 2.

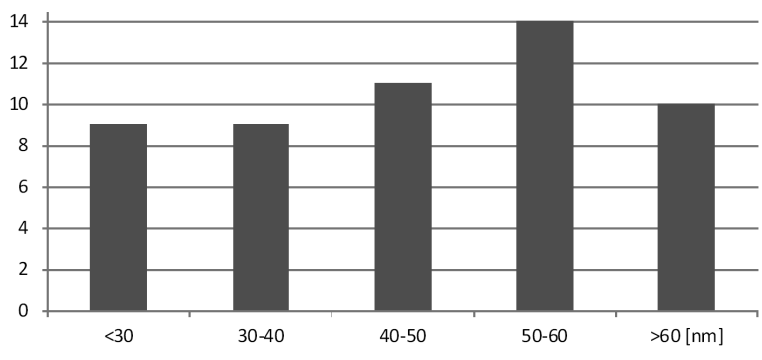

Fig. 2. A histogram of the diameter values of $\mathrm{Ag}_{\mathrm{col}}$ particles (set of 53 particles, data extracted from 18 AFM topography images).

\subsection{SERS activity of Ag colloidal solutions and deposited films}

At first the spectrum of CV $\left(10^{-4} \mathrm{M}\right)$ was recorded (see Fig. 3, black line), then mixed with the $\mathrm{Ag}_{\text {col }}$ solutions and the spectrum was recorded again (CV concentration in the sample equal to $5 \times 10^{-6} \mathrm{M}$ ). The AEF was calculated separately for two Raman active modes of $\mathrm{CV}$ :

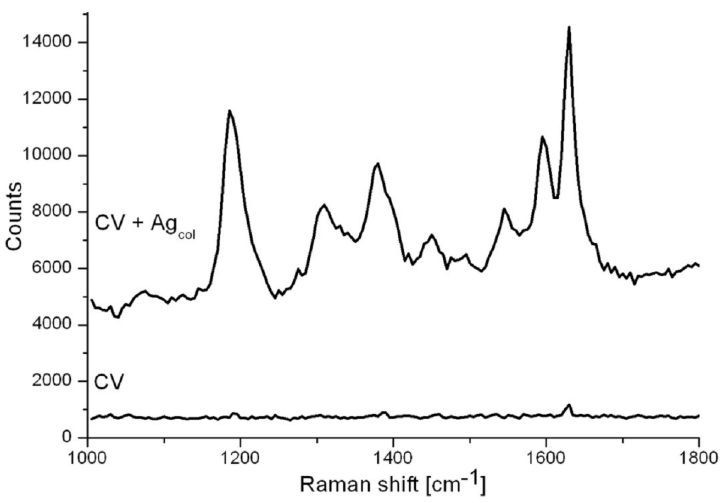

Fig. 3. Raman spectra of $\mathrm{CV}\left(\mathrm{RS}, C_{\mathrm{RS}}=10^{-4} \mathrm{M}\right)$ and a mixture of $\mathrm{CV}$ and $\mathrm{Ag}_{\text {col }}$ (SERRS, $c_{\mathrm{SERS}}=5 \times$ $\left.10^{-6} \mathrm{M}\right)$.

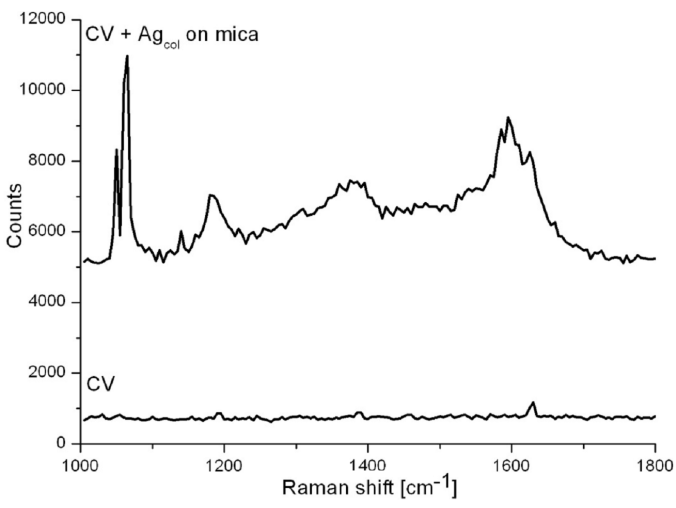

Fig. 4. Raman spectra of CV (RS, $\left.C_{\mathrm{RS}}=10^{-4} \mathrm{M}\right)$ and a mixture of $\mathrm{CV}$ and $\mathrm{Ag}_{\text {col }}$ deposited on mica. 
$1177(1200) \mathrm{cm}^{-1}$ and $1621 \mathrm{~cm}^{-1}$. For a mixture of $\mathrm{CV}$ and $\mathrm{Ag}_{\text {col }}$ the $\mathrm{AEF}_{1177}=133$ and the $\mathrm{AEF}_{1621}=290$. For the $\mathrm{CV}$ and $\mathrm{Ag}_{\text {col }}$ mixtures deposited on mica the Raman spectrum was recorded (see Fig. 4) and compared with the spectrum of $\mathrm{CV}$. The AEF values are equal to $\mathrm{AEF}_{1177}=160$ and the $\mathrm{AEF}_{1621}=82$.

\section{Discussion and conclusion}

The obtained results of AFM imaging show that the $\mathrm{Ag}_{\text {col }}$ particles are not uniform in size, however their mean diameter is close to the nominal value. The variety of the diameter can be caused by either the clustering in the solution or AFM experiments conditions. For this reason imaging of 2 samples and 3 areas were performed and statistical methods applied to determine the diameter of the particles. The enhancement obtained in the SERS experiments is not as high as reported in the literature, however one should remember that the experiments were conducted using an open light path spectrometer, not a microscope. The results confirmed that both, $\mathrm{Ag}_{\text {col }}$ solutions and films exhibit enhancing properties and can be used as a SERS active substrate.

\section{Acknowledgments}

A.G. and A.B. would like to acknowledge financial support from the Faculty of Physics, Astronomy and Applied Informatics of the Nicolaus Copernicus University through a Ph.D. scholarship and statutory activities grant (task no. 101).

\section{References}

[1] M. Moskovits, Rev. Mod. Phys. 57, 783 (1985).

[2] K. Kneipp, H. Kneipp, in: Surface-Enhanced Raman Scattering: Physics and Applications; Eds. K. Kneipp, H. Kneipp, M. Moskovits; Topics in Applied Physics, Springer, Berlin 2006, Vol. 103, p. 183.

[3] K. Kneipp, Y. Wang, H. Kneipp, L.T. Perelman, I. Itzkan, R.R. Dasari, M.S. Feld, Phys. Rev. Lett. 78, 1667 (1997).

[4] J.D. Jiang, E. Burstein, H. Kobayashi, Phys. Rev. Lett. 57, 1793 (1986).

[5] K. Hering, D. Cialla, K. Ackermann, T. Dorfer, R. Moller, H. Schneidewind, R. Mattheis, W. Fritzsche, P. Rosch, J. Popp, Anal. Bioanal. Chem. 390, 113 (2008).

[6] R.C. Le Ru, E. Blackie, M. Meyer, P.G. Etchegoin, J. Phys. Chem. C 111, 13794 (2007).

[7] L. Bujak, D. Piątkowski, S. Mackowski, S. Wörmke, C. Jung, C. Bräuchle, A. Agarwal, N.A. Kotov, T. Schulte, E. Hofmann, T.H.P. Brotosudarmo, H. Scheer, A.O. Govorov, R.G. Hiller, Acta Phys. Pol. A 116, S-22 (2009).

[8] P.C. Lee, D.J. Meisel, J. Phys. Chem. 86, 3391 (1982). 